\title{
Observed Medical Device Type Not Present In Intended Location Type
}

National Cancer Institute

\section{Source}

National Cancer Institute. Observed Medical Device Type Not Present In Intended

Location Type. NCI Thesaurus. Code C147153.

A description of the type of medical device that is found in a subject but is not present in its intended location within the body. 